\title{
ISB News January 2012: From the Editor-in-Chief
}

\section{Scott Sheridan}

(C) ISB 2011

One of the keys to the continued success of the International Journal of Biometeorology is the quality, commitment, and hard work of its Field Editors. I would like to take this opportunity to announce a number of important changes to the IJB Field Editor structure and personnel. After a few months of transition, I am happy to report that the editorial board structure is now complete for the next 3-year period. We have expanded the Field Editor ranks from 9 to 12 , through five additions and two retirements. Retiring are Nigel A. S. Taylor and Richard de Dear. We thank both Nigel and Richard for their work on the Journal over many years. Our new members are:

- Lynda Chambers, The Centre for Australian Weather and Climate Research, Bureau of Meteorology, Australia

- Grady Dixon, Department of Geosciences, Mississippi State University, USA

- Yoram Epstein, Heller Institute of Medical Research, Tel Aviv University, Israel

- Thomas Holst, Department of Earth and Ecosystem Sciences, Lund University, Sweden

- Kim Knowlton, Health \& Environment Program, Natural Resources Defense Council, USA

Through the creation of some new Field Editor titles and minor shifts in some other Field Editor titles, it is expected we can overall better cover the current spectrum of submissions to IJB. The full list of Field Editor titles and Field Editors is now:

\section{Climate Change and Ecology}

Lynda Chambers

\section{Epidemiology I}

Grady Dixon

\section{S. Sheridan $(\square)$}

Department of Geography, Kent State University,

Kent, Ohio 44242, USA

e-mail: ssherid1@kent.edu
Climate Change and Public Health

Kristie L. Ebi

\section{Humans I}

Yoram Epstein

\section{Humans II}

Masaaki Hashimoto

Forests

Thomas Holst

Epidemiology II

Kim Knowlton

Thermal Environment

Christina Koppe

\section{Animals}

Nicola Lacetera

\section{Urban Biometeorology}

Helmut Meyer

\section{Agriculture}

Simone Orlandini

\section{Phenology}

Mark D. Schwartz

You will of course see this new Field Editor line-up on the following final pages of each issue of the Journal, including this one, complete with further contact details of each Field Editor. 